\title{
Spin-Triplet Pairing Induced by Hund's Rule Exchange in Orbitally Degenerate Systems: Hartree-Fock Approximation
}

\author{
M. ZEGRODNIK ${ }^{a, *}$ AND J. SPAŁEK ${ }^{a, b}$ \\ ${ }^{a}$ AGH University of Science and Technology, Faculty of Physics and Applied Computer Science \\ al. A. Mickiewicza 30, 30-059 Krakow, Poland \\ ${ }^{b}$ Marian Smoluchowski Institute of Physics, Jagiellonian University \\ W.S. Reymonta 4, 30-059 Kraków, Poland
}

\begin{abstract}
We discuss the spin-triplet pairing mechanism induced by the Hund rule ferromagnetic exchange. We include explicitly the effect of interband hybridization and treat the problem by starting from an extended Hubbard model for a doubly degenerate band, making the simplest Hartree-Fock approximation for the part involving the pairing and the Hubbard interaction. The conditions of stability of various phases are determined as a function of both band filling and microscopic parameters. The phase diagram contains regions of stability of the spin-triplet superconducting phase coexisting with either saturated or non-saturated ferromagnetism. Phase diagrams for the cases of constant density of states and that of square lattice have been provided. The influence of hybridization on the stability of considered phases, as well as the temperature dependences of magnetic moment and the superconducting gap are also discussed.
\end{abstract}

PACS: 74.20.-z, 74.25.Dw, 75.10.Lp

\section{Introduction}

The candidates for the spin-triplet superconductors have been discovered in the last two decades. They are $\mathrm{Sr}_{2} \mathrm{RuO}_{4}$ [1], $\mathrm{UGe}_{2}$ [2], and URhGe [3]. Particularly interesting are the last two as the paired state appears inside the ferromagnetic phase and, in the case of $\mathrm{UGe}_{2}$, disappears at the critical pressure together with ferromagnetism.

The question we posed for the first time a decade ago [4-7] was whether the two phenomena may have the same origin - the intra-atomic ferromagnetic Hund rule exchange. This question originated from drawing an analogy between the present systems and cuprate high-temperature superconductors where the antiferromagnetic kinetic exchange is often regarded as the source of both antiferromagnetism (in parent Mott-Hubbard insulating compound) and spin-singlet superconductivity when the metallic state is stabilized by doping the insulator with holes. Namely, if the exchange interaction induced superconductivity is a reasonable mechanism, not just an accident, one has to explore other possibilities such as the ferromagnetic interaction.

One should note a principal limitation for the exchange interaction to represent a feasible mechanism of pairing, which takes place in direct space. Namely, this is the pairing induced by interparticle exchange. Therefore one

\footnotetext{
* corresponding author; e-mail: michal.zegrodnik@gmail.com
}

may think that the hopping (bare band) energy has to become comparable to the exchange-interaction strength. This idea is tested in the present paper in the simplest Hartree-Fock approximation. It turns out that even in the weak-coupling limit, the paired state (the so-called A phase) may appear below the Stoner threshold for the onset of ferromagnetism, as well as coexist with it above that threshold (in the form of A1 phase). The result is independent of the form of the density of states taken and when interband hybridization is included.

\section{Theoretical model}

We consider the extended orbitally degenerate Hubbard Hamiltonian, which has the form

$$
\begin{aligned}
\hat{H} & =\sum_{i j(i \neq j) l \sigma} t_{i j} a_{i l \sigma}^{\dagger} a_{j l \sigma}+\sum_{i j(i \neq j) l l^{\prime}\left(l \neq l^{\prime}\right) \sigma} t_{i j}^{12} a_{i l \sigma}^{\dagger} a_{j l^{\prime} \sigma} \\
& +U \sum_{i l} \hat{n}_{i l \uparrow} \hat{n}_{i l \downarrow}-J \sum_{i l l^{\prime}\left(l \neq l^{\prime}\right)}\left(\hat{\boldsymbol{S}}_{i l} \cdot \hat{\boldsymbol{S}}_{i l^{\prime}}+\frac{3}{4} \hat{n}_{i l} \hat{n}_{i l^{\prime}}\right),
\end{aligned}
$$

where $l=1,2$ and the first term describes electron hopping between atomic sites $i$ and $j$. The second term introduces hybridization into the system. The third term describes the Coulomb interaction between electrons on the same orbital. The fourth term introduces the Hund rule ferromagnetic exchange between electrons localized on the same site, but on different orbitals. In this model we neglect the interaction-induced intra-atomic singlet-pair 
hopping $\sim J$ and the correlation induced hopping [8], as we deal with the triplet-paired and ferromagnetic phases only. It can be shown that [5] one can represent the full exchange term with the help of the real-space pair operators, i.e.,

$$
J \sum_{i l l^{\prime}\left(l \neq l^{\prime}\right)}\left(\hat{\boldsymbol{S}}_{i l} \cdot \hat{\boldsymbol{S}}_{i l^{\prime}}+\frac{3}{4} \hat{n}_{i l} \hat{n}_{i l^{\prime}}\right) \equiv 2 J \sum_{i, m} \hat{A}_{i m}^{\dagger} \hat{A}_{i m}
$$

which are defined in the following way [9, 10]:

$$
\hat{A}_{i, m}^{\dagger} \equiv \begin{cases}a_{i 1 \uparrow}^{\dagger} a_{i 2 \uparrow}^{\dagger}, & m=1, \\ a_{i 1 \downarrow}^{\dagger} a_{i 2 \downarrow}^{\dagger}, & m=-1, \\ \frac{1}{\sqrt{2}}\left(a_{i 1 \uparrow}^{\dagger} a_{i 2 \downarrow}^{\dagger}+a_{i 1 \downarrow}^{\dagger} a_{i 2 \uparrow}^{\dagger}\right), & m=0 .\end{cases}
$$

Hamiltonian (1) cannot be treated rigorously, apart from model situations $[11,12]$. In present paper we treat the pairing and the Hubbard part in the mean-field approximation. Effectively, after making the Hartree-Fock approximation we can write down the Hamiltonian in the following way (in reciprocal space):

$$
\begin{aligned}
& \hat{H}_{\mathrm{HF}}=\sum_{\boldsymbol{k l \sigma}}\left(\epsilon_{\boldsymbol{k}}-\mu-\sigma I S^{z}\right) \hat{n}_{\boldsymbol{k} l \sigma} \\
& +\sum_{\boldsymbol{k} l l^{\prime}\left(l \neq l^{\prime}\right) \sigma} \epsilon_{12 \boldsymbol{k}} a_{\boldsymbol{k} l \sigma}^{\dagger} a_{\boldsymbol{k} l^{\prime} \sigma} \\
& +\sum_{\boldsymbol{k}, m= \pm 1}\left(\Delta_{m}^{*} \hat{A}_{\boldsymbol{k}, m}+\Delta_{m} \hat{A}_{\boldsymbol{k}, m}^{\dagger}\right) \\
& +\sqrt{2} \sum_{\boldsymbol{k}}\left(\Delta_{0}^{*} \hat{A}_{\boldsymbol{k}, 0}+\Delta_{0} \hat{A}_{\boldsymbol{k}, 0}^{\dagger}\right) \\
& +N\left[\frac{\left|\Delta_{1}\right|^{2}+\left|\Delta_{-1}\right|^{2}+2\left|\Delta_{0}\right|^{2}}{2 J}\right. \\
& \left.+\frac{n^{2}}{2}(3 J-U)+2 I\left(S^{z}\right)^{2}\right],
\end{aligned}
$$

where $I=U+J$ is the effective magnetic coupling constant, $N$ is the number of atomic sites and $\epsilon_{\boldsymbol{k} 1}=\epsilon_{\boldsymbol{k} 2} \equiv \epsilon_{\boldsymbol{k}}$ is the dispersion relation in the doubly degenerate band. For modeling purpose, we assume that the bands are identical. In the Hamiltonian written above we have introduced the superconducting spin-triplet gap parameters

$$
\Delta_{ \pm 1} \equiv-\frac{2 J}{N} \sum_{\boldsymbol{k}}\left\langle\hat{A}_{\boldsymbol{k}, \pm 1}\right\rangle, \quad \Delta_{0} \equiv-\frac{2 J}{\sqrt{2} N} \sum_{\boldsymbol{k}}\left\langle\hat{A}_{\boldsymbol{k}, 0}\right\rangle .
$$

Moreover, as the bands are identical, we assume that the corresponding band fillings and magnetic moments are also identical, i.e. $n_{1}=n_{2} \equiv n / 2$ and $S_{1}^{z}=S_{2}^{z} \equiv S^{z}$. The one-particle part of the $\mathrm{H}-\mathrm{F}$ Hamiltonian can be easily diagonalized via unitary transformation which introduces hybridized quasi-particle operators

$$
\tilde{a}_{\boldsymbol{k} 1 \sigma}=\frac{1}{\sqrt{2}}\left(a_{\boldsymbol{k} 1 \sigma}+a_{\boldsymbol{k} 2 \sigma}\right),
$$

$$
\tilde{a}_{\boldsymbol{k} 2 \sigma}=\frac{1}{\sqrt{2}}\left(-a_{\boldsymbol{k} 1 \sigma}+a_{\boldsymbol{k} 2 \sigma}\right),
$$

with new dispersion relations

$$
\begin{aligned}
& \tilde{\epsilon}_{\boldsymbol{k} 1 \sigma}=\epsilon_{\boldsymbol{k}}-\mu-\sigma I S^{z}+\left|\epsilon_{12 \boldsymbol{k}}\right|, \\
& \tilde{\epsilon}_{\boldsymbol{k} 2 \sigma}=\epsilon_{\boldsymbol{k}}-\mu-\sigma I S^{z}-\left|\epsilon_{12 \boldsymbol{k}}\right|,
\end{aligned}
$$

for $\sigma=+1,-1$ corresponding to spin orientation up and down, respectively.

In the calculations, we make a simplifying assumption that $\epsilon_{12 \boldsymbol{k}}=\beta_{\mathrm{h}} \epsilon_{\boldsymbol{k}}$, where $\beta_{\mathrm{h}} \in[0,1]$. Let us note that the two energies correspond to the antibonding and the bonding states, respectively.

A generalized Nambu-Bogolyubov-de Gennes scheme is used next in order to write down the $\mathrm{H}-\mathrm{F}$ Hamiltonian in a matrix form allowing an easy determination of its eigenvalues. With the help of composite creation operator [5] $\tilde{\boldsymbol{f}}_{\boldsymbol{k}}^{\dagger} \equiv\left(\tilde{a}_{\boldsymbol{k} 1 \uparrow}^{\dagger}, \tilde{a}_{\boldsymbol{k} 1 \downarrow}^{\dagger}, \tilde{a}_{-\boldsymbol{k} 2 \uparrow}, \tilde{a}_{-\boldsymbol{k} 2 \downarrow}\right)$, we can construct the $4 \times 4$ Hamiltonian matrix and write

$$
\hat{H}_{\mathrm{HF}}=\sum_{\boldsymbol{k}} \tilde{\boldsymbol{f}}_{\boldsymbol{k}}^{\dagger} \tilde{\boldsymbol{H}}_{\boldsymbol{k}} \tilde{\boldsymbol{f}}_{\boldsymbol{k}}+\sum_{\boldsymbol{k} \sigma} \tilde{\epsilon}_{\boldsymbol{k} 2 \sigma}+N\{\ldots\},
$$

where

$$
\tilde{\boldsymbol{H}}_{\boldsymbol{k}}=\left(\begin{array}{cccc}
\tilde{\epsilon}_{\boldsymbol{k} 1 \uparrow} & 0 & \Delta_{1} & \Delta_{0} \\
0 & \tilde{\epsilon}_{\boldsymbol{k} 1 \downarrow} & \Delta_{0} & \Delta_{-1} \\
\Delta_{1}^{*} & \Delta_{0}^{*} & -\tilde{\epsilon}_{\boldsymbol{k} 2 \uparrow} & 0 \\
\Delta_{0}^{*} & \Delta_{-1}^{*} & 0 & -\tilde{\epsilon}_{\boldsymbol{k} 2 \downarrow}
\end{array}\right),
$$

and $\tilde{\boldsymbol{f}}_{\boldsymbol{k}} \equiv\left(\tilde{\boldsymbol{f}}_{\boldsymbol{k}}^{\dagger}\right)^{\dagger}$. We limit ourselves to the case of real gap parameters $\Delta_{m}^{*}=\Delta_{m}$, and distinguish between the following superconducting phases: $\boldsymbol{A}\left(\Delta_{1} \neq 0, \Delta_{-1} \neq 0\right.$, $\left.\Delta_{0}=0\right)$ and $\boldsymbol{A} \mathbf{1}\left(\Delta_{1} \neq 0, \Delta_{-1}=0, \Delta_{0}=0\right)$. The phase labeled as $\boldsymbol{B}$, i.e. that with $\Delta_{1} \neq 0, \Delta_{-1} \neq 0, \Delta_{0} \neq 0$ is not stable within this mechanism, so it does not appear in the subsequent discussion. After making the diagonalization transformation of (10) we can write the $\mathrm{H}-\mathrm{F}$ Hamiltonian as follows:

$$
\begin{aligned}
& \hat{H}_{\mathrm{HF}}=\sum_{\boldsymbol{k} l \sigma} \lambda_{\boldsymbol{k} l \sigma} \alpha_{\boldsymbol{k} l \sigma}^{\dagger} \alpha_{\boldsymbol{k} l \sigma}+\sum_{\boldsymbol{k} \sigma}\left(\tilde{\epsilon}_{\boldsymbol{k} 2 \sigma}-\lambda_{\boldsymbol{k} 2 \sigma}\right) \\
& \quad+N\{\ldots\} .
\end{aligned}
$$

The transformed (quasi-particle) operators have the form

$$
\begin{aligned}
& \alpha_{\boldsymbol{k} 1 \uparrow}=\tilde{U}_{\boldsymbol{k}}^{(+)} \tilde{a}_{\boldsymbol{k} 1 \uparrow}+\tilde{V}_{\boldsymbol{k}}^{(+)} \tilde{a}_{-\boldsymbol{k} 2 \uparrow}^{\dagger}, \\
& \alpha_{-\boldsymbol{k} 2 \uparrow}^{\dagger}=-\tilde{V}_{\boldsymbol{k}}^{(+)} \tilde{a}_{\boldsymbol{k} 1 \uparrow}+\tilde{U}_{\boldsymbol{k}}^{(+)} \tilde{a}_{-\boldsymbol{k} 2 \uparrow}^{\dagger}, \\
& \alpha_{\boldsymbol{k} 1 \downarrow}=\tilde{U}_{\boldsymbol{k}}^{(-)} \tilde{a}_{\boldsymbol{k} 1 \downarrow}+\tilde{V}_{\boldsymbol{k}}^{(-)} \tilde{a}_{-\boldsymbol{k} 2 \downarrow}^{\dagger}, \\
& \alpha_{-\boldsymbol{k} 2 \downarrow}^{\dagger}=-\tilde{V}_{\boldsymbol{k}}^{(-)} \tilde{a}_{\boldsymbol{k} 1 \downarrow}+\tilde{U}_{\boldsymbol{k}}^{(-)} \tilde{a}_{-\boldsymbol{k} 2 \downarrow}^{\dagger},
\end{aligned}
$$

where the so-called Bogolyubov coherence factors acquire the form

$$
\tilde{U}_{\boldsymbol{k}}^{( \pm)}=\frac{1}{\sqrt{2}}\left(1+\frac{\epsilon_{\boldsymbol{k}}-\mu \mp I S^{z}}{\sqrt{\left(\epsilon_{\boldsymbol{k}}-\mu \mp I S^{z}\right)^{2}+\Delta_{ \pm 1}^{2}}}\right)^{1 / 2},
$$




$$
\tilde{V}_{\boldsymbol{k}}^{( \pm)}=\frac{1}{\sqrt{2}}\left(1-\frac{\epsilon_{\boldsymbol{k}}-\mu \mp I S^{z}}{\sqrt{\left(\epsilon_{\boldsymbol{k}}-\mu \mp I S^{z}\right)^{2}+\Delta_{ \pm 1}^{2}}}\right)^{1 / 2} .
$$

The dispersion relations for the quasi-particles $\lambda_{\boldsymbol{k} l \sigma}$ are then

$$
\begin{aligned}
& \lambda_{\boldsymbol{k} 1 \uparrow}=\sqrt{\left(\epsilon_{\boldsymbol{k}}-\mu-I S^{z}\right)^{2}+\Delta_{1}^{2}}+\beta_{\mathrm{h}}\left|\epsilon_{\boldsymbol{k}}\right|, \\
& \lambda_{\boldsymbol{k} 1 \downarrow}=\sqrt{\left(\epsilon_{\boldsymbol{k}}-\mu+I S^{z}\right)^{2}+\Delta_{-1}^{2}}+\beta_{\mathrm{h}}\left|\epsilon_{\boldsymbol{k}}\right|, \\
& \lambda_{\boldsymbol{k} 2 \uparrow}=\sqrt{\left(\epsilon_{\boldsymbol{k}}-\mu-I S^{z}\right)^{2}+\Delta_{1}^{2}}-\beta_{\mathrm{h}}\left|\epsilon_{\boldsymbol{k}}\right|, \\
& \lambda_{\boldsymbol{k} 2 \downarrow}=\sqrt{\left(\epsilon_{\boldsymbol{k}}-\mu+I S^{z}\right)^{2}+\Delta_{-1}^{2}}-\beta_{\mathrm{h}}\left|\epsilon_{\boldsymbol{k}}\right| .
\end{aligned}
$$

Defining the gap parameters as $\Delta_{ \pm 1}$, the average number of particles per atomic site as $n=\sum_{l}\left\langle\hat{n}_{i l \uparrow}+\hat{n}_{i l \downarrow}\right\rangle$, and the average magnetic moment per band per site as $S^{z}=\left\langle\hat{n}_{i l \uparrow}-\hat{n}_{i l \downarrow}\right\rangle / 2$, the set of self-consistent equations can be constructed for calculating all unknown mean-field parameters

$$
\begin{aligned}
& 1=-J \int_{-1 / 2}^{1 / 2} \frac{\rho(\epsilon)}{\sqrt{\left(\epsilon-\mu-I S^{z}\right)^{2}+\Delta_{1}^{2}}} \\
& \times\left(f\left(\lambda_{1 \uparrow}\right)+f\left(\lambda_{2 \uparrow}\right)-1\right) \mathrm{d} \epsilon, \\
& 1=-J \int_{-1 / 2}^{1 / 2} \frac{\rho(\epsilon)}{\sqrt{\left(\epsilon-\mu+I S^{z}\right)^{2}+\Delta_{-1}^{2}}} \\
& \times\left(f\left(\lambda_{1 \downarrow}\right)+f\left(\lambda_{2 \downarrow}\right)-1\right) \mathrm{d} \epsilon, \\
& n-2=\int_{-1 / 2}^{1 / 2} \rho(\epsilon)\left\{\frac{\epsilon-\mu-I S^{z}}{\sqrt{\left(\epsilon-\mu-I S^{z}\right)^{2}+\Delta_{1}^{2}}}\right. \\
& \times\left(f\left(\lambda_{1 \uparrow}\right)+f\left(\lambda_{2 \uparrow}\right)-1\right)+\frac{\epsilon-\mu+I S^{z}}{\sqrt{\left(\epsilon-\mu+I S^{z}\right)^{2}+\Delta_{-1}^{2}}} \\
& \left.\times\left(f\left(\lambda_{1 \downarrow}\right)+f\left(\lambda_{2 \downarrow}\right)-1\right)\right\} \mathrm{d} \epsilon \\
& S^{z}=\frac{1}{4} \int_{-1 / 2}^{1 / 2} \rho(\epsilon)\left[\frac{\epsilon-\mu-I S^{z}}{\sqrt{\left(\epsilon-\mu-I S^{z}\right)^{2}+\Delta_{1}^{2}}}\right. \\
& \times\left(f\left(\lambda_{1 \uparrow}\right)+f\left(\lambda_{2 \uparrow}\right)-1\right)-\frac{\epsilon-\mu+I S^{z}}{\sqrt{\left(\epsilon-\mu+I S^{z}\right)^{2}+\Delta_{-1}^{2}}} \\
& \left.\times\left(f\left(\lambda_{1 \downarrow}\right)+f\left(\lambda_{2 \downarrow}\right)-1\right)\right] \mathrm{d} \epsilon,
\end{aligned}
$$

where $\rho(\epsilon)$ is the bare density of states (per site per spin per orbital) and we have normalized all energies to the bare bandwidth, $W$, while $f(x)$ stands for the FermiDirac distribution function. Using Eq. (11) we can calculate the free energy functional per site of the system

$$
\frac{F}{N}=-T \int_{-1 / 2}^{1 / 2} \rho(\epsilon) \sum_{l \sigma} \ln \left(1+\exp \left(-\frac{\lambda_{l \sigma}}{T}\right)\right) \mathrm{d} \epsilon
$$

$$
+\int_{-1 / 2}^{1 / 2} \rho(\epsilon) \sum_{\sigma}\left(\tilde{\epsilon}_{2 \sigma}+\lambda_{2 \sigma}\right) \mathrm{d} \epsilon+\mu n+\{\ldots\},
$$

where $T \equiv k_{\mathrm{B}} T / W$ denotes the reduced temperature. Substituting the solutions for $\Delta_{ \pm 1}, \mu$ and $S^{z}$ coming from Eqs. (15)-(18) to Eq. (19), we obtain the physical free energy which determine relative stability of different magnetic and/or paired states which we discuss next.

\section{Results and discussion}

Phase diagrams on the plane $(n, J)$ have been calculated for the case of constant density of states in the starting (non-hybridized) bands and for that appropriate for the square lattice. In both cases, regions of stability of the following six different phases have been determined and involve the states: NS - normal state, A - superconducting phase A, SFM - saturated ferromagnet, $\mathrm{FM}$ - nonsaturated ferromagnet, $\mathrm{A} 1+\mathrm{FM}$ - coexisting superconducting phase A1 and FM phase, A1+SFM coexisting superconducting phases A1 and SFM phase. Exemplary phase diagrams for selected parameters are shown in Fig. 1 for the constant density of states and for

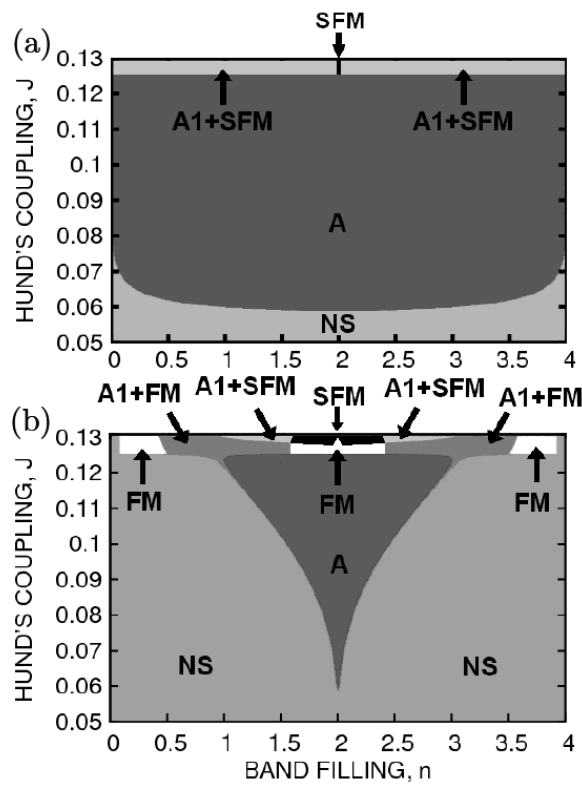

Fig. 1. Phase diagrams for $T=10^{-4}$ for the case of constant density of states in the starting (non-hybridized) bands for $\beta_{\mathrm{h}}=0.0$ (a) and $\beta_{\mathrm{h}}=0.04(\mathrm{~b})$. The Coulomb repulsion constant $U=7$ J. No antiferromagnetism was included here.

hybridization parameter $\beta_{\mathrm{h}}=0$ (a) and $\beta_{\mathrm{h}}=0.04$ (b). The presence of hybridization enriches remarkably the phase diagram. Let us note that the Stoner threshold is reached for $J=0.125$; only above this critical value the coexistent $(\mathrm{S}) \mathrm{FM}+\mathrm{A} 1$ phase appears. One has to emphasize that the pairing is induced by the exchange, not by any spin (moment) fluctuation. This is clearly seen from the fact that it can appear even in the ferromagnetically saturated state. The same type of phase diagrams 

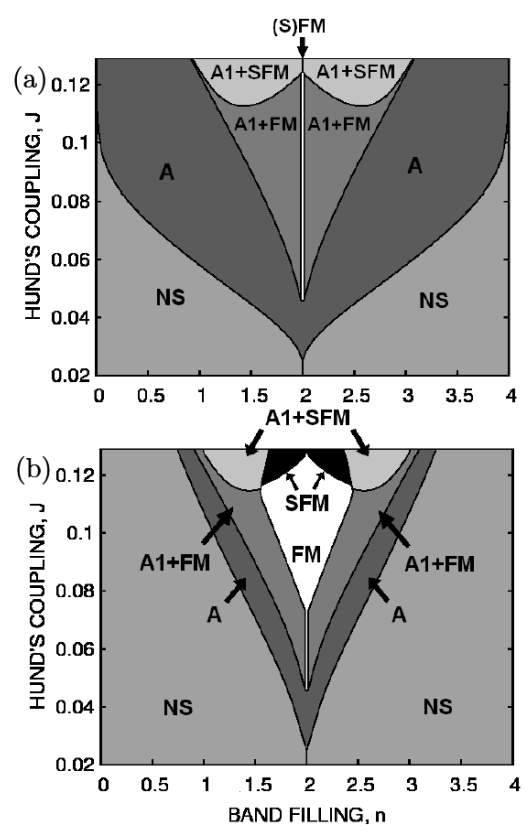

Fig. 2. Phase diagrams for $T=10^{-4}$ for the case of square lattice for $\beta_{\mathrm{h}}=0.0$ (a) and $\beta_{\mathrm{h}}=0.04(\mathrm{~b})$. The Coulomb repulsion constant $U=7 \mathrm{~J}$. No antiferromagnetism was included here.

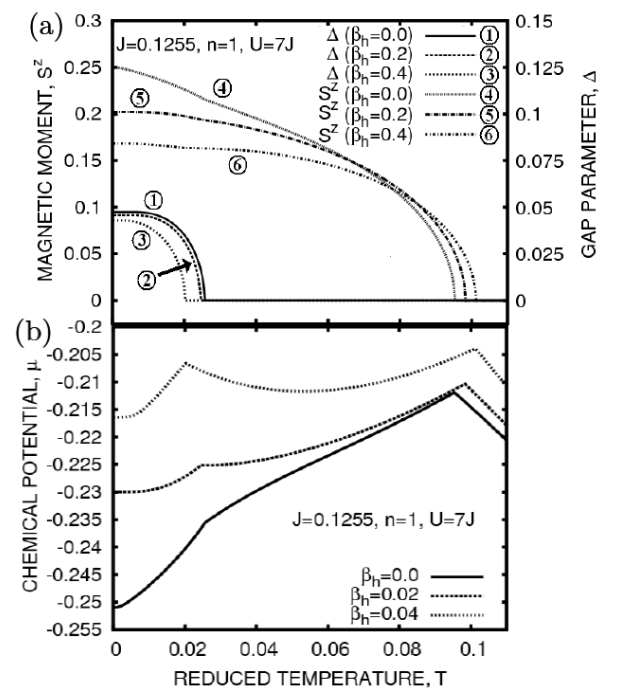

Fig. 3. Temperature dependence of the superconducting gap $\Delta \equiv \Delta_{ \pm 1}$ and magnetic moment $S^{z}$ (a), as well as the chemical potential $\mu$ (b), for selected values of $\beta_{\mathrm{h}}$ parameter. Figures correspond to a stable coexisting A1+(S)FM phase for the density of states in the starting (non-hybridized) bands appropriate for the square lattice.

but for a square lattice (with $W=8|t|$ ) are exhibited in Fig. 2 .

It can be seen in the presented diagrams that the stronger is the hybridization parameter the smaller are the regions of stability of the superconducting phases for both density-of-states functions considered. One has to note that these phase diagrams are still incomplete. This is because near the half-filling one can expect the ap- pearance of stable antiferromagnetic phases [13]. Therefore, the regime of superconductivity stability may be restricted to even narrower strips on the phase diagrams. On the other hand, because of the peaked nature of the density of states in both cases, ferromagnetism may compete successfully with antiferromagnetism even close to $n \approx 2$. This question requires certainly further studies. One should notice that the A $1+\mathrm{FM}$ phase disappears for $n=2$ even for the case where there is no hybridization, i.e., only the SFM phase survives then.

For the sake of completness, we display in Fig. 3 the thermodynamic quantities $S^{z}, \Delta \equiv \Delta_{ \pm 1}$, and $\mu$, all versus temperature, for selected values of $\beta_{\mathrm{h}}$. The values of microscopic parameters $n$ and $J$ have been chosen so that the $\mathrm{A} 1+(\mathrm{S}) \mathrm{FM}$ phase would be the stable one. As the temperature of considered system is rising one can observe two phase transitions. The strength of hybridization influences the temperatures in which phase transitions occur. The increase of $\beta_{\mathrm{h}}$ parameter results in diminution of the critical temperature, $T_{\mathrm{S}}$, and increase of the Curie temperature, $T_{\mathrm{C}}$. What is more the hybridization makes the magnetic moment smaller in low temperatures. Let us note that the transition temperature ratio $T_{\mathrm{C}} / T_{\mathrm{S}} \approx 5$.

\section{Conclusions}

We have carried out the Hartree-Fock analysis of the phase diagrams involving the ferromagnetic and spin-triplet superconducting phases within the extended two-band degenerate Hubbard model. Stable and coexisting superconducting and ferromagnetic phases have been obtained. We have analyzed in detail the influence of inter-band hybridization on the stability of these phases, as well as have provided the temperature dependence of the order parameters. For particular set of microscopic parameters, one observes two separate phase transitions as a function of temperature. The first is the transition from $\mathrm{A} 1+\mathrm{FM}$ phase to $\mathrm{FM}$ at $T_{\mathrm{S}}$ and the second one is from $\mathrm{FM}$ to NS at $T_{\mathrm{C}} \sim 5 T_{\mathrm{S}}$. According to our results, the hybridization has a negative influence on the spin-triplet superconductivity, as it decreases the critical temperature and reduces the regime of stability of the superconducting phases. On the contrary, $T_{\mathrm{C}}$ increases with the increasing hybridization parameter $\beta_{\mathrm{h}}$. The phase diagrams determined by us supplement the corresponding magnetic phase diagrams [13] with the A and coexistent A1+FM, A1+SFM phases for theoretical model of the same class.

\section{Acknowledgments}

M.Z. has been partly supported by the EU Human Capital Operation Program, Polish Project No. POKL.04.0101-00-434/08-00. J.S. acknowledges the financial support of the Foundation for Polish Science (FNP) within project TEAM. The authors thanks also for the support of the Ministry of Science and Higher Education through grant No. N N 202128736. 


\section{References}

[1] Y. Maeno, H. Hashimoto, K. Yoshida, S. Nishizaki, T. Fujita, J.G. Bednorz, Nature 372, 532 (1994).

[2] S.S. Saxena, P. Agarwal, K. Ahilan, F. M. Grosche, R.K.W. Haselwimmer, M.J. Steiner, E. Pugh, I. R. Walker, S.R. Julian, P. Monthoux, G.G. Lonzarich, A. Huxley, I. Sheikin, D. Braithwaite, J. Flouquet, Nature 406, 587 (2000).

[3] N. Tateiwa, T.C. Kobayashi, K. Hanazono, K. Amaya, Y. Haga, R. Settai, Y. Onuki, J. Phys., Condens. Matter 13, 117 (2001).

[4] For additional introduction see: J. Spałek, Acta Phys. Pol. A 111, 409 (2007).

[5] K. Klejnberg, J. Spałek, J. Phys., Condens. Matter 11, 6553 (1999).
[6] J. Spałek, Phys. Rev. B 63, 104513 (2001).

[7] K. Klejnberg, J. Spałek, Phys. Rev. B 61, 15542 (2000).

[8] T. Nomura, K. Yamada, J. Phys. Soc. Jpn. 71, 1993 (2002).

[9] J. Spałek, P. Wróbel, W. Wójcik, Physica C 387, 1 (2003).

[10] P. Wróbel, Ph.D. Thesis, Jagiellonian University, Kraków 2004, unpublished.

[11] Shun-Qing Shen, Phys. Rev. B 57, 6474 (1998).

[12] J. Dukelsky, C. Esebagg, S. Pittel, Phys. Rev. Lett. 88, 062501 (2002).

[13] S. Inagaki, R. Kubo, Int. J. Magn. 4, 139 (1973). 